\title{
Mental practice in isolation improves cervical joint position sense in patients with chronic neck pain: A randomized single- blind placebo trial
}

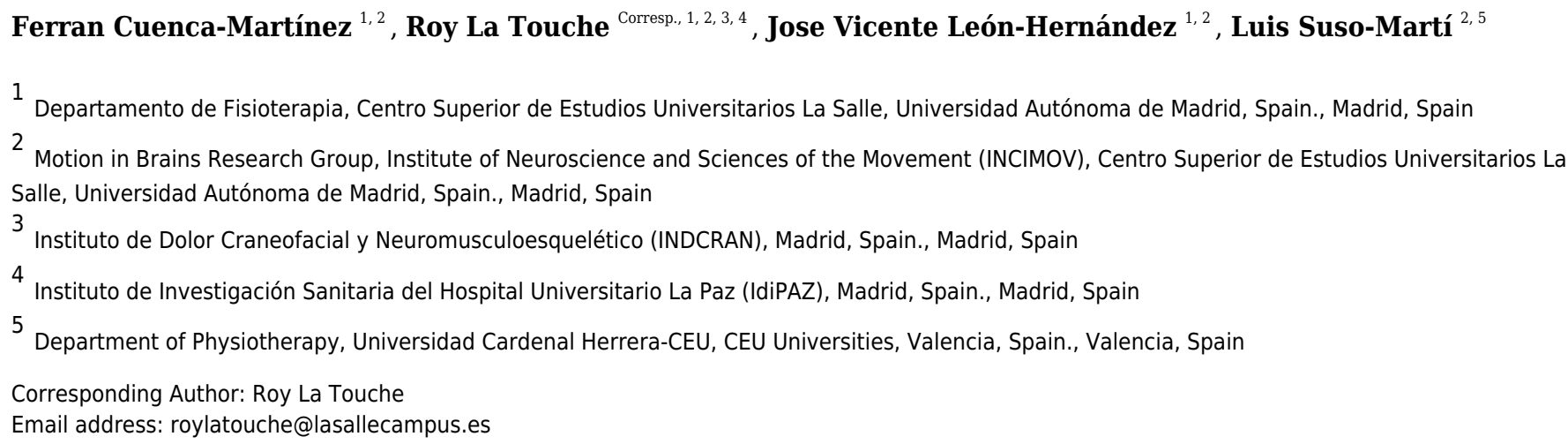

Objective: The main objective of this trial was to assess whether action observation (AO) training and motor imagery (MI) produced changes in the cervical joint position sense (CJPS) both at the end of the intervention and 10 minutes postintervention compared with a placebo intervention in patients with nonspecific chronic neck pain (NSCNP). Methods: A single-blind placebo clinical trial was designed. A total of 30 patients with NSCNP were randomly assigned to the $\mathrm{AO}$ group, $\mathrm{MI}$ group or placebo observation (PO) group. CJPS in flexion, extension and rotation movements in both planes were the main variables.

Results: The results obtained in the vertical plane showed that the AO group obtained greater improvements than the PO group in the CJPS in terms of cervical extension movement both at the end of the intervention and 10 minutes postintervention $(p=.001$, $d=1.81$ and $p=.004, d=1.74$, respectively), and also in cervical flexion movement, although only at 10 minutes after the intervention $(p=.035, d=0.72)$. In addition, the $A O$ group obtained greater improvements than the MI group in the CJPS only at the end of the intervention in cervical extension movement ( $p=.041, d=1.17$ ). Regarding the left rotation cervical movement, both the $\mathrm{MI}$ and $\mathrm{AO}$ groups were superior to the $\mathrm{PO}$ group in both planes at the end of the intervention $(p<.05, d>0.80)$. Conclusions: Although both $\mathrm{AO}$ and $\mathrm{MI}$ could be a useful strategy for CJPS improvement, the AO group showed the strongest results. The therapeutic potential of the application of mental practice in a clinical context in the early stages of rehabilitation of NSCNP should be considered. 


\section{Mental practice in isolation improves cervical joint position sense in patients}

\section{2 with chronic neck pain: A randomized single-blind placebo trial}

3 Running title: Mental practice in chronic pain

4 Ferran Cuenca-Martínez ${ }^{\mathrm{a}, \mathrm{b}}$, Roy La Touche, ${ }^{\mathrm{a}, \mathrm{b}, \mathrm{c}, \mathrm{d}, *}$, Jose Vicente León-Hernández ${ }^{\mathrm{a}, \mathrm{b}}$ and Luis

5 Suso-Martíb,e

6 a Departamento de Fisioterapia, Centro Superior de Estudios Universitarios La Salle, Universidad 7 Autónoma de Madrid, Spain.

8 b Motion in Brains Research Group, Institute of Neuroscience and Sciences of the Movement 9 (INCIMOV), Centro Superior de Estudios Universitarios La Salle, Universidad Autónoma de Madrid, 10 Spain.

$11{ }^{\mathrm{c}}$ Instituto de Dolor Craneofacial y Neuromusculoesquelético (INDCRAN), Madrid, Spain.

12 d Instituto de Investigación Sanitaria del Hospital Universitario La Paz (IdiPAZ), Madrid, Spain.

13 e Department of Physiotherapy, Universidad Cardenal Herrera-CEU, CEU Universities, Valencia, 14 Spain.

*Corresponding author. Address: Facultad de Ciencias de la Salud, Centro Superior de Estudios 80, Fax: (+34) 913571730 E-Mail: roylatouche@yahoo.es 


\section{Abstract}

Objective: The main objective of this trial was to assess whether action observation (AO) training and motor imagery (MI) produced changes in the cervical joint position sense (CJPS) both at the end of the intervention and 10 minutes postintervention compared with a placebo intervention in patients with nonspecific chronic neck pain (NSCNP). Methods: A single-blind placebo clinical trial was designed. A total of 30 patients with NSCNP were randomly assigned to the AO group, MI group or placebo observation (PO) group. CJPS in flexion, extension and rotation movements in both planes were the main variables. Results: The results obtained in the vertical plane showed that the AO group obtained greater improvements than the PO group in the CJPS in terms of cervical extension movement both at the end of the intervention and 10 minutes postintervention ( $p=.001, d=1.81$ and $p=.004, d=1.74$, respectively), and also in cervical flexion movement, although only at 10 minutes after the intervention $(p=.035, d=0.72)$. In addition, the AO group obtained greater improvements than the MI group in the CJPS only at the end of the intervention in cervical extension movement $(p=.041, d=1.17)$. Regarding the left rotation cervical movement, both the $\mathrm{MI}$ and $\mathrm{AO}$ groups were superior to the PO group in both planes at the end of the intervention $(p<.05, d>0.80)$. Conclusions: Although both AO and MI could be a useful strategy for CJPS improvement, the AO group showed the strongest results. The therapeutic potential of the application of mental practice in a clinical context in the early stages of rehabilitation of NSCNP should be considered.

Keywords: Action observation, Motor imagery, Joint position sense, Chronic neck pain. 


\section{Introduction}

Neck pain is a common musculoskeletal disorder with a high prevalence in the population and is the fourth leading disability-generating condition (Vos et al., 2013). Chronic neck pain is often considered nonspecific (NSCNP), due to the difficulty in identifying the origin of the pain, when imaging tests provide no relevant information for establishing an accurate pathological diagnosis (Bogduk, 2011). This clinical condition is thought to have a multidimensional nature due to the combination of a complex pathogenesis, the presence of maladaptive processes of central neuroplasticity and in pain processing, as well as the relevance of psychological aspects involved in the NSCNP such as anxiety or pain catastrophism (Binder, 2007; Dimitriadis et al., 2015; Muñoz-García et al., 2016).

It is commonly reported that patients with NSCNP present an alteration in the cervical joint position (Alahmari et al., 2017). The cervical region has a large number of proprioceptive receptors, especially in the upper cervical spine (Falla, Jull, \& Hodges, 2004; Falla, Bilenkij, \& Jull, 2004). It has been suggested that in patients with NSCNP, proprioceptive afferent information from the cervical spine might be impaired due to the presence of chronic pain (Uremović et al., 2007). In addition, Kim, Kim, \& Nabekura (2017) have suggested that patients with persistent pain might undergo a process of maladaptive neuroplasticity in major sensitive areas such as the primary somatosensory area. Furthermore, Hodges \& Tucker (2011) suggested that maladaptive processes of central plasticity could lead to impaired motor planning and movement execution as a pain response and could therefore affect motor control and movement acuity in this region.

To improve proprioceptive input in the cervical region, several interventions have been proposed, including craniocervical motor control exercises (MCEs) (Izquierdo et al., 2016; Kim \& Kwag, 2016; Lee \& Kim, 2016). MCEs have been shown to reduce pain and disability in patients with NSCNP compared with other types of treatments (Martin-Gomez et al., 2019). However, it has been suggested that patterns of muscle activation and recruitment are altered in the presence of pain, so implementing MCEs is a challenging aspect in these patients and might lead patients to perform them incorrectly, which could reduce their effectiveness (Sterling et al., 2001a). One of the alternatives in those early stages of intervention could be mental practice based on mental motor imagery (MI) and action observation (AO). 
71 MI is defined as a dynamic mental process that involves the representation of an action, in an

72 internal manner, without its actual motor execution (Decety, 1996). AO evokes an internal, real-

73 time simulation of what the observer is seeing (Buccino, 2014). It has been shown that both MI

74 and AO training can activate neurocognitive mechanisms underlying the planning and execution

75 of voluntary movements in a similar manner as when this movement is actually performed

76 (Wright, Williams, \& Holmes, 2014). Previously Villafañe et al. (2016) found an improvement

77 in motor function through mental practice after total hip arthroplasty.

78 The authors hypothesize that both forms of mental practice in isolation could lead to changes in cervical joint position sense (CJPS) compared with a placebo intervention. Therefore, the main objective of the present study was to assess whether AO training and mental MI produced changes in CJPS both at the end of the intervention and 10 minutes postintervention in

82 comparison with a placebo intervention in patients with NSCNP.

\section{Methods}

\section{Study design}

The present study was a randomized, single-blind placebo clinical trial, planned and conducted in accordance with Consolidated Standards of Reporting Trials requirements (Schulz, Altman,

87 Moher, \& CONSORT Group, 2010) (Figure 1) and was approved by La Salle University Center

88 for Higher Education (CSEULS-PI-027/2019).

89 This study was registered in the United States Randomized Trials Registry on clinicaltrial.gov

90 (trial registry number: NCT03910829). All patients completed the informed consent document

91 prior to the study.

\section{Recruitment of participants}


93 Patients who were diagnosed by their family doctor as having NSCNP were referred to the

94 primary care physiotherapy service, and all met the inclusion criteria of the study at one

95 physiotherapy center. Participants were recruited between April 2019 and May 2019.

96 The inclusion criteria were as follows: (a) men and women aged between 18 and 65 years; and

97 (b) a medical diagnosis of NSCNP with at least 6 months of neck pain symptoms. Exclusion

98 criteria were the following: (a) patients with rheumatic diseases, cervical hernia or radicular pain,

99 cervical whiplash syndrome, neck surgeries or a history of arthrodesis; (b) systemic diseases; (c)

100 vision, hearing or vestibular problems; and (d) severe trauma or a traffic accident that had an

101 impact on the cervical area. All the participants were given an explanation of the study

102 procedures, which were planned under the ethical standards of the Helsinki Declaration. In

103 addition, this randomized, single-blind placebo clinical trial was used as a pilot with the aim of

104 calculating the sample size of a future study.

\section{Randomization}

106 Randomization was performed using a computer-generated random sequence table with a non-

107 balanced 3-block design (GraphPad Software, Inc., CA, USA). An independent researcher

108 generated the randomization list, and a member of the research team who was not involved in the

109 assessment or intervention of the participants was in charge of the randomization and maintained

110 the list. Those included were randomly assigned to 1 of the 3 groups using the random-sequence

111 list, ensuring concealed allocation.

\section{Blinding}


113 The assessments and treatments were performed by different therapists. The evaluator was

114 blinded to the participant's assignment. All the intervention procedures were performed by the

115 same physiotherapist, who had more than three years of experience in the field and was blinded

116 to the purpose of the study. Patients were blinded to their group allocation.

\section{Interventions}

118 The interventions were previously described by Suso-Martí, León-Hernández, La Touche, Paris-

119 Alemany, \& Cuenca-Martínez (2019).

120 Action observation group

121 Patients in this group performed an exclusive AO protocol of 2 commonly used MCEs in the

122 treatment of patients with NSCNP (Jull, Falla, Vicenzino, \& Hodges, 2009; Jull, Falla,

123 Treleaven, Hodges, \& Vicenzino, 2007; O’Leary, Falla, Hodges, Jull, \& Vicenzino, 2007). Both

124 exercises were based on the motor gesture of craniocervical flexion (Figure 2). Patients in the

125 AO group performed the observation by watching a video of the continuous performance of both

126 exercises repeatedly during 2 series of 1 minute for each exercise, with a total duration of 4

127 minutes.

128 The first exercise consisted of maintaining the cervical spine in a neutral position in a sitting

129 position and performing a deep muscle contraction to flatten the curve of the neck, nodding with

130 the head. The second exercise involved a deep muscle contraction by performing the

131 craniocervical flex-extension gesture with the resistance of an elastic band.

132 Motor imagery group

133 The patients in this group performed an MI protocol of the same cervical exercises as the AO

134 group (Figure 2). Patients were instructed on the movements they were to imagine by showing

135 both exercises and the precise instructions for each movement. After this, they were instructed to 
136 perform a third-person mental task of visual MI of both exercises during 2 series of 1 minute for

137 each exercise, with a total duration of 4 minutes.

138 Placebo observation group

139 Patients in the placebo observation (PO) group underwent a placebo AO protocol. The patients

140 watched during the same intervention time as both previous groups. This documentary video was

141 composed of video clips of nature landscapes, without any human agent or motor gesture.

142 This type of placebo AO protocol has been used in previous studies (Bang, Shin, Kim, \& Choi,

143 2013; Giovanni Buccino et al., 2012).

144 Outcomes

145 Primary outcomes

146 Cervical joint position sense

147 CJPS is an objective measure of neck repositioning sense and can quantify the alteration in neck 148 proprioception. CJPS was assessed with a visual feedback device, the SenMoCOR LED (Sensory 149 Motor Control-Oriented Rehabilitation, IAOM-US). This device consists of adjustable straps and 150 a fastening support for a laser beam. It is adjustable to the evaluator's desired position, allowing 151 projection of the light beam.

152 The experimental procedure for assessing CJPS with a laser beam has been described by Revel, 153 Andre-Deshays, \& Minguet (1991). First, patients were asked to sit in a comfortable position at 154 90-cm distance from the bullseye with the SenMoCOR Kit correctly placed. With eyes closed, 155 they were asked to point to the neutral position of the head and memorize it to return to after the 156 completion of the movement. This point was recorded as a reference for each patient. The patient 157 subsequently performed a maximal movement of cervical flexion and then attempted to find the 158 initial reference position with a maximum of accuracy without speed instructions. The point on 
159 which the light beam stopped indicated the global error measured in centimeters $(\mathrm{cm})$ in relation

160 to the center of the target recorded previously. The assessor measured the deviations from de

161 target position for each trial on both axes (x/y). Values of $\mathrm{x}$ (abscissa) and $\mathrm{y}$ (ordinate) were

162 recorded according to the Cartesian coordinate system. The same protocol was used for the

163 extension, right and left rotation movements. Ten trials were performed with head repositioning

164 after each movement, and the mean measure was recorded. No feedback was given to the

165 participants about their actual performance. This CJPS testing method offers an easy, quick and

166 inexpensive method for measuring cervical joint position sense. The test presents inter-rater

167 reliability ranging from moderate to good/substantial agreement (intraclass correlation

168 coefficient $[\mathrm{ICC}] \geq 0.51-0.75$ ); it also presents intra-rater reliability ranging from moderate to

169 almost perfect agreement (ICC $\geq 0.48-0.82$ ) (Juul, Langberg, Enoch, \& Søgaard, 2013). The

170 minimal detectable change ranged from $0.52-0.75 \mathrm{~cm}$ (Juul et al., 2013).

171 Secondary outcomes

172 Ability to generate mental motor imagery

173 The movement imagery questionnaire-revised (MIQ-R) is an 8-item self-report inventory that

174 was used to assess visual and kinesthetic motor imagery ability. Four different movements are

175 included in the MIQ-R, which is comprised of 4 visual and 4 kinesthetic items. For each item,

176 participants read a description of the movement. They then physically performed the movement

177 and were instructed to reassume the starting position after finishing the movement and before

178 performing the mental task, imagining the movement visually or kinesthetically. Each participant

179 then rated the ease or difficulty of mentally generating that image on a 7-point scale, in which 7

180 indicates "very easy to see/feel" and 1 "very difficult to see/feel." The internal consistencies of

181 the MIQ-R have been consistently adequate, with Cronbach's $\alpha$ coefficients ranging above 0.84

Peer] reviewing PDF | (2019:07:39644:1:1:NEW 8 Aug 2019) 
182 for the total scale, 0.80 for the visual subscale and 0.84 for the kinesthetic subscale (Campos \&

183 González, 2010).

184 Mental chronometry

185 Mental chronometry (MC) is a reliable measure that has been widely used to record objective 186 measurements of the ability to create mental motor images (Guillot \& Collet, 2005; Malouin,

187 Richards, Durand, \& Doyon, 2008; Williams, Guillot, Di Rienzo, \& Cumming, 2015). To assess $188 \mathrm{MC}$, first, the time dedicated to imagining each task was recorded using a stopwatch. The time 189 between the interval command to start the task (given by the evaluator) and the verbal response 190 at the conclusion of the task (given by the participant) was recorded. After the MI task, the 191 participants were asked to perform the real movement execution of the task, and the time 192 dedicated to performing each task was recorded using a stopwatch. Both time measurements 193 were taken to obtain the temporal congruence between the tasks. In healthy participants, for the 194 temporal congruence test, the ICC ranged from $0.63-0.95$, whereas the ICC for intrasession 195 reliability ranged from 0.95-0.97 (Malouin et al., 2008).

196

197 198

\section{Pain-related fear of movement}

Pain-related fear of movement was assessed using the 11-item Spanish version of the Tampa Scale of kinesiophobia, whose reliability and validity have been demonstrated (Gómez-Pérez, López-Martínez, \& Ruiz-Párraga, 2011). The Tampa scale for Kinesiophobia consists of 2 subscales, one related to fear of activity and the other related to fear of harm. The final score can range between 11 and 44 points, with higher scores indicating greater perceived kinesiophobia (Gómez-Pérez et al., 2011). Internal consistency ratings were moderate. In the chronic pain sample, Cronbach's $\alpha=0.79$ was obtained using total TSK items (Gómez-Pérez et al., 2011). 
204 Neck disability

205 Disability was measured using the Spanish-validated Neck Disability Index (NDI), which

206 consists of 10 items related to daily functional activities. Each question is measured on a scale

207 from 0 (no disability) to 5, and an overall score out of 100 is calculated by adding each item

208 score together and multiplying it by 2 . A higher NDI score indicates greater perceived disability

209 due to neck pain. It has been shown to have high "test-retest" reliability and to have appropriate

210 psychometric properties (Alfonso Andrade Ortega, Damián Delgado Martínez, \& Almécija Ruiz,

211 2008). MacDermid et al. (2009) concluded that differences in 7 points out of 50 in the NDI

212 should be considered as clinically relevant. In addition, intraclass correlation coefficients (ICCs)

213 ranged from 0.50 to 0.98 .

\section{Level of physical activity}

215 The level of physical activity was assessed using the IPAQ questionnaire, which allows the

216 participants to be divided into 3 groups according to their level of activity, which can be high,

217 moderate, and low or inactive (Roman-Viñas et al., 2010). This questionnaire has shown

218 acceptable validity and psychometric properties to measure total physical activity. Therefore, the

219 psychometric properties of the questionnaire were accepted for use in studies that required the

220 measurement of physical activity; reliability was approximately $0.65(\mathrm{r}=0.76$; 95\% CI $0.73-$

221 0.77) (Mantilla Toloza \& Gómez-Conesa, 2007).

\section{Procedures}

223 Data were collected as previously described by Suso-Martí et al. (2019). Each participant was

224 given an informed consent document to participate in the study, in addition to a set of 
225 questionnaires to complete before starting the intervention. These questionnaires included

226 psychometrics forms and a questionnaire about age, gender, time with pain duration and pain

227 intensity. The psychological variables were evaluated with self-assessments. Then MIQ-R and

228 mental chronometry were assessed, and the pre-intervention measurements of CJPS were then

229 taken. Subsequently, in a sitting position, patients performed the AO, MI or PO protocol,

230 according to their group. Immediately after the intervention, a blinded evaluator measured the

231 CJPS in the 4 movements. Following this, patients were asked to sit and relax comfortably,

232 without movement, for 10 minutes, and the CJPS was measured again (post 2).

\section{Statistical analysis}

234 The statistical analysis was performed using SPSS software version 22.0 (SPSS Inc., Chicago,

235 IL, USA).

236 The normality of the variables was evaluated using the Shapiro-Wilk test. Descriptive statistics

237 were used to summarize the data for the continuous variables and are presented as mean \pm

238 standard deviation and 95\% confidence interval. A two-way repeated measures analysis of

239 variance (ANOVA) was conducted to study the effect of the between-subject factor 'intervention

240 group' with 3 categories (i.e., AO, MI and PO) and the within-subject called 'time' with also 3

241 categories (i.e., pre, post, and post 2) on the dependent variables. Partial eta squared $\left(\eta_{p}{ }^{2}\right)$ was

242 calculated as a measure of effect size (strength of association) for each main effect and

243 interaction in the ANOVAs, with $0.01-0.059$ representing a small effect, $0.06-0.139$ a medium

244 effect and $>0.14$ a large effect (Cohen, 1973). A post hoc analysis with Bonferroni correction

245 was performed in the case of significant ANOVA findings for multiple comparisons between

246 variables. Effect sizes $(d)$ were calculated according to Cohen's method, in which the magnitude

247 of the effect was classified as small $(0.20-0.49)$, moderate $(0.50-0.79)$ or large $(0.8)$ (Cohen, 
248 1988). The $\alpha$ level was set at .05 for all tests. Additionally, we compared age, weight and height

249 between groups, to explore whether the groups were homogeneous at baseline with a 1-factor 250 ANOVA.

\section{Results}

252 A total of 30 patients with NSCNP were included and were randomly allocated into 3 groups of

25310 participants per group. There were no adverse events reported in either group. No statistically 254 significant differences in demographic data were present preintervention between the groups and 255 the self-report variables (Table 1).

\section{Flexion range of motion}

257 X-plane

258 Regarding the flexion range of motion (ROM) in the X-plane, the ANOVA revealed significant 259 changes in group $*$ time $\left(F=4.06, p=.006, \eta_{\mathrm{p}}^{2}=0.231\right)$ and time $\left(F=17.45, p<.001, \eta_{\mathrm{p}}^{2}=\right.$ 260 0.393). The post hoc analysis revealed significant within-group differences in both the MI and 261 AO groups, with a moderate-large effect size for the MI group at postintervention $(p<.001, d=$ $2620.95)$ and at post 2 intervention $(p=.021, d=0.72)$, as well as with a moderate-large effect size 263 for $\mathrm{AO}$ at postintervention $(p<.001, d=0.96)$ and at post 2 intervention $(p=.001, d=0.74)$.

264 The post hoc analysis revealed no significant within-group differences in the PO group $(p>.05)$

265 (Table 2). However, no significant differences were found between the groups $(p>.05)$.

266 Y-plane

267 Regarding the flexion ROM in the Y-plane, the ANOVA revealed significant changes in 268 group*time $\left(F=4.14, p=.005, \mathrm{\eta}_{\mathrm{p}}^{2}=0.235\right)$ and time $\left(F=8.83, p<.001, \mathrm{\eta}_{\mathrm{p}}{ }^{2}=0.246\right)$. The post $269 h o c$ analysis revealed significant within-group differences only in the AO group, with a 270 moderate-large effect size at postintervention $(p<.001, d=1.01)$ and at post 2 intervention $(p=$ $271.005, d=0.77)$. The post hoc analysis revealed no significant within-group differences in the PO 
272 or MI groups ( $p>.05$ ) (Table 2). Regarding the between groups comparison, only the AO group

273 showed significant differences with the PO group at post 2 intervention, with a moderate effect

274 size $(p=.035, d=0.72)$ (Figure 3).

275 Extension range of motion

276 X-plane

277 Regarding the extension ROM in the X-plane, there were no significant differences in time $(F=$

$\left.2781.87, p=.16, \mathrm{\eta}_{\mathrm{p}}^{2}=0.065\right)$ or in group*time $\left(F=0.33, p=.33, \mathrm{\eta}_{\mathrm{p}}^{2}=0.024\right)$.

$279 \quad$ Y-lane

280 Regarding the extension ROM in the Y-plane, the ANOVA revealed significant changes in

281 group*time $\left(F=6.87, p<.001, \mathrm{\eta}_{\mathrm{p}}{ }^{2}=0.337\right)$ and time $\left(F=8.56, p=.001, \mathrm{\eta}_{\mathrm{p}}{ }^{2}=0.241\right)$. The post

$282 h o c$ analysis revealed significant within-group differences in both the MI and AO groups, with a

283 moderate-large effect size for MI at postintervention $(p=.017, d=0.77)$ and at post 2

284 intervention $(p=.007, d=1.04)$, as well as with a large effect size for AO at postintervention $(p$

$285<.001, d=1.01)$ and at post 2 intervention $(p=.006, d=1.05)$. The post hoc analysis revealed

286 no significant within-group differences in the PO group $(p>.05)$ (Table 3).

287 Regarding the between groups comparison, the AO group showed significant differences with

288 both the MI and the PO groups at postintervention, with a large effect size $(p=.041, d=1.17$,

289 and $p=.001, d=1.81$, respectively) and at post 2 intervention with only the PO group, with a

290 large effect size $(p=.004, d=1.74)$ (Figure 3).

291 Left rotation range of motion

292 X-plane

293 Regarding the left rotation ROM in the X-plane, the ANOVA revealed significant changes in

294 group*time $\left(F=3.08, p=.023, \eta_{\mathrm{p}}{ }^{2}=0.186\right)$ but not in time $\left(F=1.53, p=.226, \mathrm{\eta}_{\mathrm{p}}{ }^{2}=0.054\right)$. 
295 The post hoc analysis revealed no significant within-group differences in any group $(p>.05)$

296 (Table 4). However, both the MI and AO groups showed significant between group differences

297 with the PO group at postintervention, with a large effect size $(p=.035, d=1.29$, and $p=.005$,

$298 d=1.54$, respectively) (Figure 4).

299 Y-plane

300 Regarding the left rotation ROM in the Y-plane, the ANOVA revealed significant changes in

301 group*time $\left(F=5.44, p=.002, \mathrm{\eta}_{\mathrm{p}}^{2}=0.287\right)$ and time $\left(F=9.58, p=.001, \mathrm{\eta}_{\mathrm{p}}{ }^{2}=0.262\right)$. The post

$302 h o c$ analysis revealed significant within-group differences in both the MI and AO groups, with a

303 large effect size for MI at postintervention $(p=.012, d=1.18)$ and at post 2 intervention $(p=$

$304.009, d=0.87)$, as well as with a large effect size for AO at postintervention $(p=.004, d=1.71)$

305 and at post 2 intervention $(p=.001, d=1.25)$. The post hoc analysis revealed no significant

306 within-group differences in the PO group ( $p>.05)$ (Table 4). In addition, both the MI and AO

307 groups showed significant between group differences with the PO group at postintervention, with

308 a large effect size ( $p=.016, d=1.24$, and $p=.001, d=1.70$, respectively) (Figure 5).

309 Right rotation range of motion

310 X-plane

311 Regarding the right rotation ROM in the X-plane, the ANOVA revealed significant changes in

312 group*time $\left(F=2.81, p=.034, \eta_{\mathrm{p}}^{2}=0.172\right)$ but not in time $\left(F=1.98, p=.147, \mathrm{\eta}_{\mathrm{p}}^{2}=0.069\right)$.

313 The post hoc analysis revealed significant within-group differences in the AO group, with a

314 moderate effect size, only at postintervention $(p=.011, d=0.72)$. However, significant within-

315 group differences were also found between post-post 2 intervention in the AO group, showing a

316 loss of effect after 10 minutes $(p=.02, d=-0.61)$ (Table 5). Finally, no significant differences

317 were found between the groups $(p>.05)$ (Figure 4). 
$318 \quad$ Y-plane

319 Regarding the right rotation ROM in the Y-plane, the ANOVA revealed significant changes over

320 time $\left(F=7.53, p=.003, \eta_{\mathrm{p}}{ }^{2}=0.218\right)$ but not in group $*$ time $\left(F=1.75, p=.151, \mathrm{\eta}_{\mathrm{p}}{ }^{2}=0.115\right)$.

321 The post hoc analysis revealed significant within-group differences only in the AO group, with a

322 large effect size, at postintervention $(p=.006, d=1.24)$ and at post 2 intervention $(p=.043, d=$

323 0.94). The post hoc analysis revealed no significant within-group differences in the PO or MI

324 groups $(p>.05)$ (Table 5). Finally, no significant differences were found between the groups $(p$

$325>.05)$ (Figure 5).

\section{Sample size calculation}

327 The sample size was estimated with the program $G^{*}$ Power 3.1.7 for Windows $\left(G^{*}\right.$ Power@ from

328 University of Dusseldorf, Germany) (Faul et al., 2007). The sample size calculation was

329 considered as a power calculation to detect between-group differences in a primary outcome

330 measures (flexion movement). We considered 3 groups and 2 measurements for primary

331 outcomes to obtain $95 \%$ statistical power (1- $\beta$ error probability) with an $\alpha$ error level probability

332 of 0.05 using analysis of variance (ANOVA) of repeated measures, within-between interaction,

333 and an effect size of $\eta p 2=0.231$ obtained from our results. This generated a sample size of total

334 of 42 participants plus an estimated $15 \%$ loss in follow-up, yielding a total of 48 participants (16

335 per group).

336 Discussion

337 The main objective of the present study was to assess whether AO training and mental MI

338 produced changes in CJPS both at the end of the intervention and at 10 minutes postintervention

339 compared with a placebo intervention in patients with NSCNP. 
340 The results obtained in the vertical plane showed that the AO group obtained greater

341 improvements than the PO group in CJPS of the cervical extension movement both at the end of

342 the intervention and at 10 minutes postintervention, as well as in the cervical flexion movement,

343 although only at 10 minutes after the intervention. In addition, the AO group obtained greater

344 improvements than the MI group in CJPS only at the end of the intervention of the cervical

345 extension movement. However, in the horizontal plane of the flexo-extension movements,

346 neither of the two mental practice groups was superior to the placebo intervention.

347 On the other hand, the results obtained in the vertical plane showed that both the AO and MI

348 groups obtained greater improvements than the PO group in CJPS of the cervical left rotation

349 movement at the end of the intervention. However, no significant differences were found

350 between the groups in the right cervical rotation movement. Finally, in the horizontal plane,

351 again both the AO and MI groups obtained greater improvements than the PO group in CJPS of

352 the cervical left rotation movement at the end of the intervention, but no significant differences

353 were found in the right cervical rotation movement between the groups.

354 NSCNP usually presents an alteration in CJPS (Alahmari et al., 2017). Chronic pain could affect 355 receptors and the transmission of proprioceptive information from the cervical region, one of the 356 keys to an adequate sense of joint position (Uremović et al., 2007). In addition, impaired 357 transmission of proprioceptive information in patients with chronic musculoskeletal pain could 358 lead to neuroplastic reorganization of body schema in the primary somatosensory area (Kim et 359 al., 2017). Body schema is the model that is used by the musculoskeletal system for control, and 360 its disruption is thought to cause incongruence between motor output and proprioceptive 361 feedback (Mccormick, Zalucki, Hudson, \& Moseley, 2007). Therefore, in the current treatment 362 of patients with NSCNP, proprioceptive training has been proposed, with the aim of improving 
363 internal representation both through exercise and manual therapy, which could reduce pain and

364 disability in these patients (Treleaven, 2008). It is possible that the overlap of neural processes

365 between $\mathrm{MI}$ and $\mathrm{AO}$ with real movement execution could provoke similar effects to real 366 proprioceptive stimulation (Hardwick, Caspers, Eickhoff, \& Swinnen, 2018). Our results are 367 consistent with those obtained by Beinert, Preiss, Huber, \& Taube (2015) in which 368 improvements were also found in CJPS after imagination or observation of CJPS task. It is 369 important to note that the exercises selected in the present study were specific to cranio-cervical 370 flexo-extension movement pattern, whereas in the study mentioned above the mental practice 371 was specific to the outcome variable. There are important differences between both types of 372 exercise, since the mental practice of the exercises used in this study could lead to the learning of 373 a motor gesture used in the rehabilitation of patients with NSCNP, which could lead to a 374 difference from a clinical point of view.

375 It should be noted that the two movements observed or imagined by the patients in the present 376 study were very subtle, low-joint path, highly complex, and precise movements in cervical flexo-

377 extension, and they were the two movements exclusively performed along only the vertical

378 plane. The findings showed that the strongest improvements obtained in CJPS were in the OA 379 group in the same plane and in the same flex-extension movements. However, in the horizontal 380 plane, no differences were found between the groups. These low joint range motor gestures were 381 used because it has been found that observing full cervical movements can cause a fear response 382 associated with movements perceived as dangerous (La Touche, Pérez-González, Suso-Martí, 383 Paris-Alemany, \& Cuenca-Martínez, 2018).

384 To respond to this finding, the role of mirror neurons and their relationship with the recognition 385 of actions should be analyzed. Rizzolatti, Fogassi, \& Gallese (2001) have established the "direct- 
386

387

matching hypothesis." According to this hypothesis, the OA provokes an automatic activation in the observer of the same cerebral areas related to the planning and real execution of the observed action. Given the result of the activation of these neural substrates during the execution of the action is known, the observation allows the observer to understand what is being observed through a specific observation-execution matching mechanism. Perhaps due to the high complexity of craniocervical motor control gestures, along with the fact that only single-plane movements were observed and imagined, we hypothesize that the activation of neural substrates is related to the planning and execution of voluntary movements specific to that plane. This hypothesis would explain the improvements in the CJPS in the movements of the same plane but not in the horizontal plane. However, this hypothesis is only neurophysiological, because the patients' brain activity could not be observed directly. In addition, the AO and MI groups obtained greater improvements than the PO group in CJPS in terms of cervical left rotation movement; however, this result was not maintained 10 minutes after the intervention (post 2). There might also be a nonspecific movement plane mechanism to explain this result. Previous research has shown that mental practice provides an internal position body reference, which improves the spatiotemporal control of the position of the body in space during a dynamic movement, a critical aspect in CJPS. It has been hypothesized that both MI and AO produce better integration of motor actions due to a better internal body reference despite the absence of real movement (Papadelis, Kourtidou-Papadeli, Bamidis, \& Albani, 2007). It is possible that this better internal reference of the position of the head with respect to the body could explain the positive results obtained in the left rotation, despite the fact that the mental gestures performed were in another plane of movement. However, further research is needed on the specific and nonspecific mechanisms in motor outputs induced by AO and MI. 
409 The motor control exercises selected could influence the differences found between AO and MI

410 training. Motor control exercises are difficult to imagine due to the fact that they require motor

411 learning of difficult and precise movements. Previous research has shown that movement

412 complexity and familiarity are related to MI performance (Paris-Alemany, La Touche, Gadea-

413 Mateos, Cuenca-Martínez, \& Suso-Martí, 2019). Therefore, this intervention group might have

414 been influenced by the difficulty of the mental motor image creation of these exercises. In

415 addition, MI is less effective in people with less ability to perform it (Patterson, Hoffman,

416 Palacios, \& Jensen, 2006), and it is well known that patients with chronic pain have a decreased

417 ability to create mental motor images, which could also have affected our results (Breckenridge,

418 Ginn, Wallwork, \& McAuley, 2019). Thus, taking into account all these variables, significant

419 mental effort is required, which the patients might not have been able to achieve (Cuenca-

420 Martínez, Suso-Martí, Grande-Alonso, Paris-Alemany, \& La Touche, 2018; Decety, Jeannerod,

421 Germain, \& Pastene, 1991). Regarding mental practice intervention duration, a meta-analysis by

422 Driskell et al. (1994) proposed a MI intervention for approximately $20 \mathrm{~min}$ is ideal to obtain the

423 maximum benefit from MI. Therefore, Hinshaw (1991) suggest that MI duration from 10 to 15

424 min was required for the optimal effect on performance. Perhaps the short intervention time was

425 insufficient to solve these challenges. In addition, González-Rosa et al. (2015) have shown that

426 AO provokes greater activation of cortical areas during motor learning and induces better motor

427 learning results in comparison with MI. Taube et al. (2015) showed that cortical activity was

428 higher during the combination of $\mathrm{AO}$ and $\mathrm{MI}$, so it is possible that best training effects should be 429 expected when participants apply MI during AO.

430 Moreover, previous studies have shown that AO could activate in a more ecological way the

431 mirror neuron system in front of the MI (Gatti et al., 2013). The reason for this difference is that 
432 the ventral premotor cortex receives visual inputs and could be more activated by actual visual

433 input than by the absence of visual input or overt movement, as in the case of motor imagery

434 (Rizzolatti \& Luppino, 2001). In addition, during AO, the observer has a model who performs

435 the action and in the correct context. In contrast, during motor imagery, the individual must

436 rehearse the relevant motor representations and covertly perform the action, and this could be

437 especially relevant in subjects with diminished imagery ability, as mentioned above (Gatti et al.,

438 2013; Mulder, 2007). This could lead to better motor learning via AO of new and highly

439 complex tasks (Mulder, Zijlstra, Zijlstra, \& Hochstenbach, 2004), such as those included in the

440 exercises in this study.

441 Finally, it is important to note that other therapeutic options have been used to improve cervical

442 motor control in patients with NSCNP. For example, Martin-Rodriguez et al. (2019) recently

443 found that dry needling both inside and outside the myofascial trigger point in the

444 sternocleidomastoid muscle led to improvements in cervical motor control. In addition, Sterling

445 et al. (2001b) found that spinal manual therapy provoked a decreased activity of the superficial

446 flexor muscle of the neck in a cervical motor control test. Therefore, in future studies, it would

447 be interesting to compare the effect of mental practice against or even in combination with these

448 therapeutic options.

449 Clinical implications

450 The high prevalence of patients with chronic pain, and especially with NSCNP, makes it one of

451 the most relevant musculoskeletal disorders in the rehabilitation sciences (Vos et al., 2013). It is

452 therefore essential to develop new approaches to rehabilitation strategies. In this regard, motor

453 control exercises have been shown to decrease pain and disability in patients with NSCNP

454 compared with other types of treatment (Martin-Gomez et al., 2019). However, the clinical 
455 implementation of this type of exercise in a clinical context is challenging, due to its high

456 complexity or the pain itself leading patients to not perform it or to perform it incorrectly,

457 reducing its therapeutic potential. In this regard, both MI and AO provide a simple, clinically

458 therapeutic alternative at low cost that can be performed independently by the patient. The

459 results of this study suggest that mental practice can be a useful therapeutic strategy in patients

460 with NSCNP, especially in the early stages of rehabilitation, and both strategies should be

461 considered for patients with NSCNP.

\section{Limitations}

463 This study presents several limitations. First, the sample size is small; thus, the results should be

464 considered with caution. In addition, the results have only been considered in the short term, and

465 the duration and type of intervention might be insufficient for greater increases in CJPS in

466 patients with NSCNP. Hinshaw (1991) have found that the optimal time for MI to provide the

467 greatest benefits is between 10 and 15 minutes. In the present study, the duration of the MI

468 intervention was 4 minutes. This length of time might not be sufficient to obtain the full potential

469 of MI. Further research is needed to determine the role of mental practice in the rehabilitation

470 process of patients with NSCNP.

\section{Conclusions}

472 The results obtained in the present study showed that the AO group obtained greater

473 improvements than the PO group in CJPS for the cervical extension movement both at the end of

474 the intervention and 10 minutes postintervention, as well as in the cervical flexion movement,

475 although only at 10 minutes postintervention. In addition, the AO group obtained greater

476 improvements than the MI group in the CJPS only at the end of the intervention in the cervical 
477 extension movement. Finally, regarding the left rotation cervical movement, both $\mathrm{MI}$ and AO

478 were superior to PO in both planes at the end of the intervention.

479 Our results suggest that AO training is an effective sensorimotor neurotraining tool to improve

480 CJPS in the early stages of treatment. In addition, MI could also be a tool to consider using in

481 rehabilitation, but perhaps with a longer training time. The therapeutic potential of the

482 application of mental practice in a clinical context in the early stages of rehabilitation of NSCNP

483 should be considered.

\section{Conflicts of Interest Statement}

485 The authors declare that they have no conflict of interest. This study did not receive any specific 486 grant from funding agencies in the public, commercial or not-for-profit sectors. 
487

488

489

490

491

492

493

494

495

496

497

498

499

500

501

502

503

504

505

506

507

508

509

510

511

512

513

514

515

516

517

518

519

520

\section{References}

Alahmari, K. A., Reddy, R. S., Silvian, P., Ahmad, I., Nagaraj, V., \& Mahtab, M. (2017). Influence of chronic neck pain on cervical joint position error (JPE): Comparison between young and elderly subjects. Journal of Back and Musculoskeletal Rehabilitation, 30(6), 1265-1271. http://doi.org/10.3233/BMR-169630

Alfonso Andrade Ortega, J., Damián Delgado Martínez, A., \& Almécija Ruiz, R. (2008). Validación de una versión española del Índice de Discapacidad Cervical. Medicina Clínica, 130(3), 85-89. http://doi.org/10.1157/13115352

Bang, D.-H., Shin, W.-S., Kim, S.-Y., \& Choi, J.-D. (2013). The effects of action observational training on walking ability in chronic stroke patients: a double-blind randomized controlled trial. Clinical Rehabilitation, 27(12), 1118-1125. http://doi.org/10.1177/0269215513501528

Beinert, K., Preiss, S., Huber, M., \& Taube, W. (2015). Cervical joint position sense in neck pain. Immediate effects of muscle vibration versus mental training interventions: a RCT. European Journal of Physical and Rehabilitation Medicine, 51(6), 825-32.

Binder, A. I. (2007a). The diagnosis and treatment of nonspecific neck pain and whiplash. Europa Medicophysica, 43(1), 79-89. Retrieved from http://www.ncbi.nlm.nih.gov/pubmed/17369782

Bogduk, N. (2011). The anatomy and pathophysiology of neck pain. Physical Medicine and Rehabilitation Clinics of North America, 22(3), 367-82, vii. http://doi.org/10.1016/j.pmr.2011.03.008

Breckenridge, J. D., Ginn, K. A., Wallwork, S. B., \& McAuley, J. H. (2019). Do People With Chronic Musculoskeletal Pain Have Impaired Motor Imagery? A Meta-analytical Systematic Review of the Left/Right Judgment Task. The Journal of Pain, 20(2), 119-132. http://doi.org/10.1016/j.jpain.2018.07.004

Buccino, G. (2014). Action observation treatment: a novel tool in neurorehabilitation. Philosophical Transactions of the Royal Society B: Biological Sciences, 369(1644), 20130185-20130185. http://doi.org/10.1098/rstb.2013.0185

Buccino, G., Arisi, D., Gough, P., Aprile, D., Ferri, C., Serotti, L., ... Fazzi, E. (2012). Improving upper limb motor functions through action observation treatment: a pilot study in children with cerebral palsy. Developmental Medicine \& Child Neurology, 54(9), 822-828. http://doi.org/10.1111/j.1469-8749.2012.04334.x

Campos, A., \& González, M. Á. (2010). Spanish version of the revised movement image questionnaire (MIQ-R): Psychometric properties and validation, 19(2), 265-275. 
521 Cohen, J. (1973). Eta-Squared and Partial Eta-Squared in Fixed Factor Anova Designs.

522 Educational and Psychological Measurement, 33(1), 107-112.

$523 \quad$ http://doi.org/10.1177/001316447303300111

524

525

526

527

528

529

530

531

532

533

534

535

536

537

538

539

540

541

542

543

544

545

546

547

548

549

550

551

552

553

554

555

Cohen, J. (1988). Statistical power analysis for the behavioral sciences. Statistical Power Analysis for the Behavioral Sciences. http://doi.org/10.1234/12345678

Cuenca-Martínez, F., Suso-Martí, L., Grande-Alonso, M., Paris-Alemany, A., \& La Touche, R. (2018). Combining motor imagery with action observation training does not lead to a greater autonomic nervous system response than motor imagery alone during simple and functional movements: a randomized controlled trial. PeerJ, 6, e5142. http://doi.org/10.7717/peerj.5142

Decety, J. (1996). The neurophysiological basis of motor imagery. Behav Brain Res., 77(1-2), 45-52.

Decety, J., Jeannerod, M., Germain, M., \& Pastene, J. (1991). Vegetative response during imagined movement is proportional to mental effort. Behavioural Brain Research, 42(1), 15. Retrieved from http://www.ncbi.nlm.nih.gov/pubmed/2029340

Dimitriadis, Z., Kapreli, E., Strimpakos, N., \& Oldham, J. (2015). Do psychological states associate with pain and disability in chronic neck pain patients? Journal of Back and Musculoskeletal Rehabilitation, 28(4), 797-802. http://doi.org/10.3233/BMR-150587

Driskell, J. E., Copper, C., \& Moran, A. (1994). Does mental practice enhance performance? Journal of Applied Psychology, 79(4), 481-492. http://dx.doi.org/10.1037/00219010.79.4.481

Falla, D., Bilenkij, G., \& Jull, G. (2004). Patients with chronic neck pain demonstrate altered patterns of muscle activation during performance of a functional upper limb task. Spine, 29(13), 1436-40. Retrieved from http://www.ncbi.nlm.nih.gov/pubmed/15223935

Falla, D., Jull, G., \& Hodges, P. W. (2004). Feedforward activity of the cervical flexor muscles during voluntary arm movements is delayed in chronic neck pain. Experimental Brain Research, 157(1), 43-48. http://doi.org/10.1007/s00221-003-1814-9

Faul, F., Erdfelder, E., Lang, A.-G., \& Buchner, A. (2007). G*Power: A flexible statistical power analysis program for the social, behavioral, and biomedical sciences. Behavior Research Methods, 39(2), 175-191. http://doi.org/10.3758/BF03193146

Gatti, R., Tettamanti, A., Gough, P. M., Riboldi, E., Marinoni, L., \& Buccino, G. (2013). Action observation versus motor imagery in learning a complex motor task: A short review of literature and a kinematics study. Neuroscience Letters, 540, 37-42. http://doi.org/10.1016/j.neulet.2012.11.039

Gómez-Pérez, L., López-Martínez, A. E., \& Ruiz-Párraga, G. T. (2011). Psychometric Properties 
556

557

558

559

560

561

562

563

564

565

566

567

568

569

570

571

572

573

574

575

576

577

578

579

580

581

582

583

584

585

586

587

588

589

590

of the Spanish Version of the Tampa Scale for Kinesiophobia (TSK). The Journal of Pain : Official Journal of the American Pain Society, 12(4), 425-35.

http://doi.org/10.1016/j.jpain.2010.08.004

Gonzalez-Rosa, J. J., Natali, F., Tettamanti, A., Cursi, M., Velikova, S., Comi, G., ... Leocani, L. (2015). Action observation and motor imagery in performance of complex movements: Evidence from EEG and kinematics analysis. Behavioural Brain Research, 281, 290-300. http://doi.org/10.1016/j.bbr.2014.12.016

Guillot, A., \& Collet, C. (2005). Duration of Mentally Simulated Movement: A Review. Journal of Motor Behavior, 37(1), 10-20. http://doi.org/10.3200/JMBR.37.1.10-20

Hardwick, R. M., Caspers, S., Eickhoff, S. B., \& Swinnen, S. P. (2018). Neural correlates of action: Comparing meta-analyses of imagery, observation, and execution. Neuroscience \& Biobehavioral Reviews, 94, 31-44. http://doi.org/10.1016/j.neubiorev.2018.08.003

Hinshaw, K. E. (1991). The Effects of Mental Practice on Motor Skill Performance: Critical Evaluation and Meta-Analysis. Imagination, Cognition and Personality, 11(1), 3-35. http://doi.org/10.2190/X9BA-KJ68-07AN-QMJ8

Hodges, P. W., \& Tucker, K. (2011). Moving differently in pain: A new theory to explain the adaptation to pain. Pain, 152(Supplement), S90-S98.

http://doi.org/10.1016/j.pain.2010.10.020

Izquierdo, T., Pecos-Martin, D., GirbÃ@s, E., Plaza-Manzano, G., Caldentey, R., Mel⿵̃̃ºs, R., ... Falla, D. (2016). Comparison of cranio-cervical flexion training versus cervical proprioception training in patients with chronic neck pain: A randomized controlled clinical trial. Journal of Rehabilitation Medicine, 48(1), 48-55. http://doi.org/10.2340/165019772034

Jull, G. A., Falla, D., Vicenzino, B., \& Hodges, P. W. (2009). The effect of therapeutic exercise on activation of the deep cervical flexor muscles in people with chronic neck pain. Manual Therapy, 14(6), 696-701. http://doi.org/10.1016/j.math.2009.05.004

Jull, G., Falla, D., Treleaven, J., Hodges, P., \& Vicenzino, B. (2007). Retraining cervical joint position sense: The effect of two exercise regimes. Journal of Orthopaedic Research, 25(3), 404-412. http://doi.org/10.1002/jor.20220

Juul, T., Langberg, H., Enoch, F., \& Søgaard, K. (2013). The intra- and inter-rater reliability of five clinical muscle performance tests in patients with and without neck pain. $B M C$ Musculoskeletal Disorders, 14(1), 339. http://doi.org/10.1186/1471-2474-14-339

Kim, J. Y., \& Kwag, K. Il. (2016). Clinical effects of deep cervical flexor muscle activation in patients with chronic neck pain. Journal of Physical Therapy Science, 28(1), 269-73. http://doi.org/10.1589/jpts.28.269 
591 Kim, W., Kim, S. K., \& Nabekura, J. (2017). Functional and structural plasticity in the primary 592 somatosensory cortex associated with chronic pain. Journal of Neurochemistry, 141(4), 593 499-506. http://doi.org/10.1111/jnc.14012

594

595

596

597

598

599

600

601

602

603

604

605

606

607

608

609

610

611

612

613

614

615

616

617

618

619

620

621

622

623

624

625

La Touche, R., Pérez-González, A., Suso-Martí, L., Paris-Alemany, A., \& Cuenca-Martínez, F. (2018). Observing neck movements evokes an excitatory response in the sympathetic nervous system associated with fear of movement in patients with chronic neck pain. Somatosensory \& Motor Research, 1-8. http://doi.org/10.1080/08990220.2018.1509847

Lee, K.-W., \& Kim, W.-H. (2016). Effect of thoracic manipulation and deep craniocervical flexor training on pain, mobility, strength, and disability of the neck of patients with chronic nonspecific neck pain: a randomized clinical trial. Journal of Physical Therapy Science, 28(1), 175-80. http://doi.org/10.1589/jpts.28.175

MacDermid, J. C., Walton, D. M., Avery, S., Blanchard, A., Etruw, E., McAlpine, C., \& Goldsmith, C. H. (2009). Measurement Properties of the Neck Disability Index: A Systematic Review. Journal of Orthopaedic \& Sports Physical Therapy, 39(5), 400-C12. http://doi.org/10.2519/jospt.2009.2930

Malouin, F., Richards, C. L., Durand, A., \& Doyon, J. (2008). Reliability of Mental Chronometry for Assessing Motor Imagery Ability After Stroke. Archives of Physical Medicine and Rehabilitation, 89(2), 311-319. http://doi.org/10.1016/j.apmr.2007.11.006

Mantilla Toloza, S. C., \& Gómez-Conesa, A. (2007). The International Physical Activity Questionnaire. A suitable instrument for monitoring population physical activity. Revista Iberoamericana de Fisioterapia Y Kinesiología, 10(1), 48-52. http://doi.org/10.1016/S1138-6045(07)73665-1

Martin-Gomez, C., Sestelo-Diaz, R., Carrillo-Sanjuan, V., Navarro-Santana, M. J., BardonRomero, J., \& Plaza-Manzano, G. (2019). Motor control using cranio-cervical flexion exercises versus other treatments for non-specific chronic neck pain: A systematic review and meta-analysis. Musculoskeletal Science \& Practice, 42, 52-59. http://doi.org/10.1016/j.msksp.2019.04.010

Martín-Rodríguez, A., Sáez-Olmo, E., Pecos-Martín, D., \& Calvo-Lobo, C. (2019). Effects of dry needling in the sternocleidomastoid muscle on cervical motor control in patients with neck pain: a randomised clinical trial. Acupuncture in Medicine, 37(3), 151-163. http://doi.org/10.1177/0964528419843913

Mccormick, K., Zalucki, N., Hudson, M. L., \& Moseley, G. L. (2007). Faulty proprioceptive information disrupts motor imagery : an experimental study. Australian Journal of Physiotherapy, 53(1), 41-45. http://doi.org/10.1016/S0004-9514(07)70060-0

Mulder, T. (2007). Motor imagery and action observation: cognitive tools for rehabilitation. 
626

627

628

629

630

631

632

633

634

635

636

637

638

639

640

641

642

643

644

645

646

647

648

649

650

651

652

653

654

655

656

657

658

659

660

Journal of Neural Transmission, 114(10), 1265-1278. http://doi.org/10.1007/s00702-0070763-z

Mulder, T., Zijlstra, S., Zijlstra, W., \& Hochstenbach, J. (2004). The role of motor imagery in learning a totally novel movement. Experimental Brain Research, 154(2), 211-7. http://doi.org/10.1007/s00221-003-1647-6

Muñoz-García, D., Gil-Martínez, A., López-López, A., Lopez-de-Uralde-Villanueva, I., La Touche, R., \& Fernández-Carnero, J. (2016). Chronic Neck Pain and Cervico-Craniofacial Pain Patients Express Similar Levels of Neck Pain-Related Disability, Pain Catastrophizing, and Cervical Range of Motion. Pain Research and Treatment, 2016, 7296032. http://doi.org/10.1155/2016/7296032

O’Leary, S., Falla, D., Hodges, P. W., Jull, G., \& Vicenzino, B. (2007). Specific Therapeutic Exercise of the Neck Induces Immediate Local Hypoalgesia. The Journal of Pain, 8(11), 832-839. http://doi.org/10.1016/j.jpain.2007.05.014

Papadelis, C., Kourtidou-Papadeli, C., Bamidis, P., \& Albani, M. (2007). Effects of imagery training on cognitive performance and use of physiological measures as an assessment tool of mental effort. Brain and Cognition, 64(1), 74-85. http://doi.org/10.1016/j.bandc.2007.01.001

Paris-Alemany, A., La Touche, R., Gadea-Mateos, L., Cuenca-Martínez, F., \& Suso-Martí, L. (2019). Familiarity and complexity of a movement influences motor imagery in dancers: A cross-sectional study. Scandinavian Journal of Medicine \& Science in Sports, 29(6), 897906. http://doi.org/10.1111/sms.13399

Patterson, D. R., Hoffman, H. G., Palacios, A. G., \& Jensen, M. J. (2006). Analgesic effects of posthypnotic suggestions and virtual reality distraction on thermal pain. Journal of Abnormal Psychology, 115(4), 834-841. http://doi.org/10.1037/0021-843X.115.4.834

Revel, M., Andre-Deshays, C., \& Minguet, M. (1991). Cervicocephalic kinesthetic sensibility in patients with cervical pain. Archives of Physical Medicine and Rehabilitation, 72(5), 28891.

Rizzolatti, G., Fogassi, L., \& Gallese, V. (2001). Neurophysiological mechanisms underlying the understanding and imitation of action. Nature Reviews Neuroscience, 2(9), 661-670. http://doi.org/10.1038/35090060

Rizzolatti, G., \& Luppino, G. (2001). The cortical motor system. Neuron, 31(6), 889-901.

Roman-Viñas, B., Serra-Majem, L., Hagströmer, M., Ribas-Barba, L., Sjöström, M., \& SeguraCardona, R. (2010). International Physical Activity Questionnaire: Reliability and validity in a Spanish population. European Journal of Sport Science, 10(5), 297-304. http://doi.org/10.1080/17461390903426667

Peer] reviewing PDF | (2019:07:39644:1:1:NEW 8 Aug 2019) 
661 Schulz, K. F., Altman, D. G., Moher, D., \& CONSORT Group. (2010). CONSORT 2010

662 Statement: updated guidelines for reporting parallel group randomised trials. $B M J$, $663 \quad 340(\operatorname{mar} 23$ 1), c332-c332. http://doi.org/10.1136/bmj.c332

664 Sterling, M., Jull, G., \& Wright, A. (2001a). The effect of musculoskeletal pain on motor activity 665 and control. The Journal of Pain, 2(3), 135-145. http://doi.org/10.1054/jpai.2001.19951

666 Sterling, M., Jull, G., \& Wright, A. (2001b). Cervical mobilisation: concurrent effects on pain, 667 sympathetic nervous system activity and motor activity. http://doi.org/10.1054/math.2000.0378

Suso-Martí, L., León-Hernández, J. V., La Touche, R., Paris-Alemany, A., \& Cuenca-Martínez, F. (2019). Motor Imagery and Action Observation of Specific Neck Therapeutic Exercises Induced Hypoalgesia in Patients with Chronic Neck Pain: A Randomized Single-Blind Placebo Trial. Journal of Clinical Medicine, 8(7), 1019. http://doi.org/10.3390/jcm8071019

Taube, W., Mouthon, M., Leukel, C., Hoogewoud, H.-M., Annoni, J.-M., \& Keller, M. (2015). Brain activity during observation and motor imagery of different balance tasks: An fMRI study. Cortex, 64, 102-114. http://doi.org/10.1016/j.cortex.2014.09.022

Treleaven, J. (2008). Sensorimotor disturbances in neck disorders affecting postural stability, head and eye movement control. Manual Therapy, 13(1), 2-11.

http://doi.org/10.1016/j.math.2007.06.003

Uremović, M., Cvijetić, S., Pasić, M. B., Serić, V., Vidrih, B., \& Demarin, V. (2007).

682

Villafañe, J. H., Pirali, C., Isgrò, M., Vanti, C., Buraschi, R., \& Negrini, S. (2016). Effects of

Williams, S. E., Guillot, A., Di Rienzo, F., \& Cumming, J. (2015). Comparing self-report and mental chronometry measures of motor imagery ability. European Journal of Sport Science, 15(8), 703-711. http://doi.org/10.1080/17461391.2015.1051133 facilitates corticospinal excitability. Frontiers in Human Neuroscience, 8, 951. http://doi.org/10.3389/fnhum.2014.00951 


\section{Table $\mathbf{1}$ (on next page)}

Table 1. Descriptive statistics of sociodemographic, self-reported and psychosocial data

Values are presented as mean \pm standard deviation or number (\%); MI: motor imagery; $A O$ : action observation; PO: placebo observation group; TSK: Tampa Scale of Kinesiophobia; NDI: neck disability index; IPAQ: International Physical Activity Questionnaire; MIQ-R: Movement Imagery Questionnaire-Revised; MC: mental chronometry; VAS: visual analog scale. 
1 Table 1. Descriptive statistics of sociodemographic, self-reported and psychosocial data

\begin{tabular}{|c|c|c|c|c|}
\hline Measures & $\begin{array}{c}\text { AO } \\
(n=10)\end{array}$ & $\begin{array}{c}\text { MI } \\
(n=10)\end{array}$ & $\begin{array}{c}\text { PO } \\
(n=10)\end{array}$ & $p$ value \\
\hline Age & $33.5 \pm 14.25$ & $30.6 \pm 11.53$ & $27.70 \pm 6.39$ & .520 \\
\hline Height (cm) & $171.9 \pm .80$ & $123.10 \pm .70$ & $174 \pm .40$ & .798 \\
\hline Weight (Kg) & $66.7 \pm 7.97$ & $68.70 \pm 4.8$ & $69.5 \pm 8.26$ & .672 \\
\hline VAS & $68.9 \pm 13.95$ & $75 \pm 7.73$ & $70.8 \pm 9.36$ & .437 \\
\hline Pain duration (m) & $27.9 \pm 17.99$ & $26.2 \pm 12.45$ & $17.4 \pm 10.05$ & .212 \\
\hline TSK-11 & $32.3 \pm 6$ & $33 \pm 4.85$ & $31.3 \pm 3.93$ & .633 \\
\hline NDI & $30.5 \pm 3.62$ & $29.8 \pm 3.82$ & $32.1 \pm 4.48$ & .430 \\
\hline IPAQ & $1760.6 \pm 483.51$ & $1713.85 \pm 500.3$ & $1785.7 \pm 659.17$ & .958 \\
\hline MIQ-R & $47.4 \pm 4.77$ & $47.3 \pm 7.86$ & $48 \pm 4.52$ & .960 \\
\hline MC & $3.65 \pm 3.96$ & $4.39 \pm 5.7$ & $4.71 \pm 4.52$ & .879 \\
\hline Sex & & & & .875 \\
\hline Male & $5(50)$ & $5(50)$ & $4(40)$ & \\
\hline Female & $5(50)$ & $5(50)$ & $6(60)$ & \\
\hline Educational Level & & & & .03 \\
\hline Secondary education & $3(30)$ & $5(50)$ & $0(00)$ & \\
\hline College education & $7(70)$ & $5(50)$ & $10(100)$ & \\
\hline Marital Status & & & & .136 \\
\hline Single & $7(70)$ & $3(30)$ & $5(50)$ & \\
\hline Married & $3(30)$ & $4(40)$ & $4(40)$ & \\
\hline Divorced & $0(0)$ & $3(30)$ & $1(0)$ & \\
\hline Medication & & & & .563 \\
\hline Yes & $7(70)$ & $5(50)$ & $7(70)$ & \\
\hline
\end{tabular}




\begin{tabular}{lccc}
\hline No & $3(30)$ & $5(50)$ & $3(30)$ \\
$\quad$ Pain Location & & & .530 \\
Right & $5(50)$ & $2(20)$ & $2(20)$ \\
Left & $3(30)$ & $5(50)$ & $4(40)$ \\
Both & $2(20)$ & $3(30)$ & $4(40)$
\end{tabular}

\begin{abstract}
Values are presented as mean \pm standard deviation or number (\%); MI: motor imagery; AO: action observation; PO: placebo observation group; TSK: Tampa Scale of Kinesiophobia; NDI: neck diłability index; IPAQ: International Physical Activity Questionnaire; MIQ-R: Movement Imagery Qॄestionnaire-Revised; MC: mental chronometry; VAS: visual analog scale.
\end{abstract}




\section{Table 2 (on next page)}

Table 2. Within-group differences in flexion cervical movement

${ }^{*} p<.05 ;{ }^{* *} p<.001 ; C l$ : confidence interval; SD: standard deviation; PO: placebo observation group; MI: motor imagery group; $A O$ : action observation group. 
Table 2. Within-group differences in flexion cervical movement

\begin{tabular}{|c|c|c|c|c|c|}
\hline \multirow[t]{3}{*}{ Measure } & \multirow[t]{2}{*}{ Group } & \multicolumn{3}{|c|}{ Mean \pm SD } & \multirow{2}{*}{$\begin{array}{c}\text { Mean difference }(95 \% \text { CI }) \text {; Effect size (d) } \\
\text { a) pre - post } \\
\text { b) pre - post } 2\end{array}$} \\
\hline & & Pre & Post & Post 2 & \\
\hline & $\mathrm{PO}$ & $12.0 \pm 4.3$ & $11.3 \pm 4.4$ & $12.16 \pm 6.1$ & $\begin{array}{l}\text { a) } 0.7(-2.1 \text { to } 3.5) ; d=0.16 \\
\text { b) }-0.1(-4.0 \text { to } 3.8) ; d=-0.1\end{array}$ \\
\hline \multirow[t]{2}{*}{$\begin{array}{l}\text { Flexion } \\
\text { X-Plane }\end{array}$} & MI & $14.6 \pm 6.0$ & $9.2 \pm 5.3$ & $10.14 \pm 6.4$ & $\begin{array}{l}\text { a) } 5.4^{* *}(2.5 \text { to } 8.2) ; d=0.95 \\
\text { b) } 4.4^{*}(0.5 \text { to } 8.4) ; d=0.72\end{array}$ \\
\hline & $\mathrm{AO}$ & $12.6 \pm 8.74$ & $6.7 \pm 7.3$ & $6.1 \pm 4.1$ & $\begin{array}{l}\text { a) } 5.9^{* *}(3.1 \text { to } 8.8) ; d=0.96 \\
\text { b) } 6.5^{*}(2.6 \text { to } 10.4) ; d=0.74\end{array}$ \\
\hline \multirow[t]{3}{*}{ Measure } & Group & & Mean \pm SD & & Mean difference $(95 \%$ CI); Effect size $(d)$ \\
\hline & & Pre & Post & Post 2 & b) pre - post 2 \\
\hline & $\mathrm{PO}$ & $9.9 \pm 4.3$ & $8.9 \pm 5.4$ & $10.8 \pm 4.0$ & $\begin{array}{l}\text { a) } 0.9(-1.6 \text { to } 3.4) ; d=0.20 \\
\text { b) }-0.9(-3.6 \text { to } 1.8) ; d=-0.21\end{array}$ \\
\hline \multirow[t]{2}{*}{$\begin{array}{l}\text { Flexion } \\
\text { Y-Plane }\end{array}$} & MI & $8.8 \pm 3.6$ & $7.6 \pm 4.0$ & $7.4 \pm 4.4$ & $\begin{array}{l}\text { a) } 1.2(-1.3 \text { to } 3.7) ; d=0.31 \\
\text { b) } 1.4(-1.3 \text { to } 4.1) ; d=0.34\end{array}$ \\
\hline & $\mathrm{AO}$ & $9.4 \pm 5.4$ & $4.6 \pm 3.7$ & $5.6 \pm 4.3$ & $\begin{array}{l}\text { a) } 4.7^{* *}(2.2 \text { to } 7.2) ; d=1.01 \\
\text { b) } 3.8^{*}(1.0 \text { to } 6.5) ; d=0.72\end{array}$ \\
\hline
\end{tabular}

"pz.05; ${ }^{* *} p<.001 ;$ CI: confidence interval; SD: standard deviation; PO: placebo observation group; MI: motor imagery group; AO: action observation group. 


\section{Table 3 (on next page)}

Table 3. Within-group differences in extension cervical movement

${ }^{*} p<.05 ;{ }^{* *} p<.001 ; C l$ : confidence interval; SD: standard deviation; PO: placebo observation group; MI: motor imagery group; $A O$ : action observation group. 
Table 3. Within-group differences in extension cervical movement

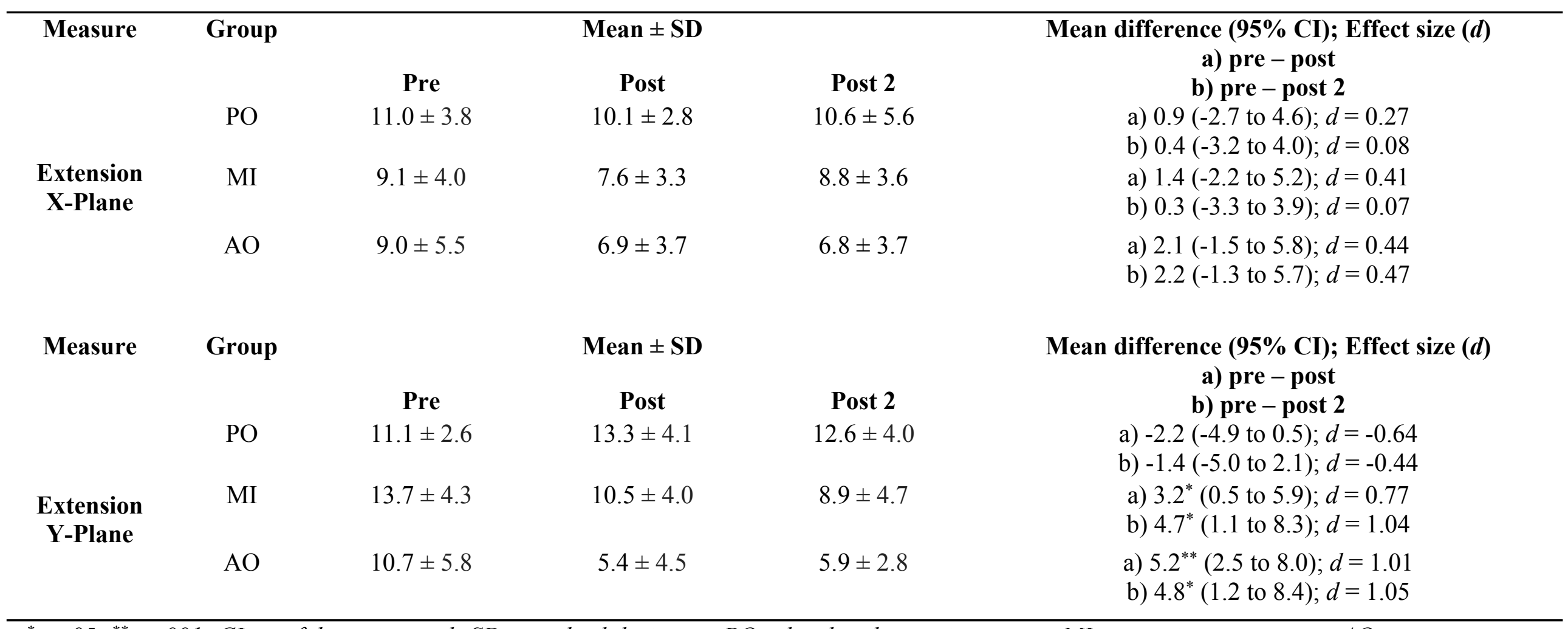

"pz.05; ${ }^{* *} p<.001 ;$ CI: confidence interval; SD: standard deviation; PO: placebo observation group; MI: motor imagery group; AO: action observation group. 


\section{Table 4 (on next page)}

Table 4. Within-group differences in left rotation cervical movement

${ }^{*} p<.05 ;{ }^{* *} p<.001 ;$ Cl: confidence interval; SD: standard deviation; PO: placebo observation group; MI: motor imagery group; $A O$ : action observation group. 
1 Table 4. Within-group differences in left rotation cervical movement

\begin{tabular}{|c|c|c|c|c|c|}
\hline Measure & \multicolumn{3}{|r|}{ Mean \pm SD } & & \multirow{2}{*}{$\begin{array}{c}\text { Mean difference }(95 \% \text { CI); Effect size }(d) \\
\text { a) pre - post } \\
\text { b) pre - post } 2\end{array}$} \\
\hline \multirow{5}{*}{$\begin{array}{l}\text { Left } \\
\text { Rotation } \\
\text { X-Plane }\end{array}$} & \multirow{3}{*}{$\mathrm{PO}$} & & Post & Post 2 & \\
\hline & & $11.0 \pm 4.8$ & $12.8 \pm 3.7$ & $12.9 \pm 3.6$ & a) $-1.8(-4.9$ to 1.2$) ; d=-0.42$ \\
\hline & & & & & b) $-1.9(-4.2$ to 0.4$) ; d=-0.44$ \\
\hline & MI & $9.3 \pm 4.1$ & $7.8 \pm 4.1$ & $8.4 \pm 3.4$ & $\begin{array}{l}\text { a) } 1.5(-1.6 \text { to } 4.6) ; d=0.36 \\
\text { b) } 0.9(-1.4 \text { to } 3.3) ; d=0.24\end{array}$ \\
\hline & $\mathrm{AO}$ & $9.3 \pm 5.3$ & $6.3 \pm 4.7$ & $8.6 \pm 4.7$ & $\begin{array}{l}\text { a) } 2.9(-0.1 \text { to } 6.0) ; d=0.59 \\
\text { b) } 0.7(-1.6 \text { to } 3.1) ; d=0.14\end{array}$ \\
\hline Measure & Group & & Mean \pm SD & & Mean difference $(95 \%$ CI $)$; Effect size $(d)$ \\
\hline \multirow{4}{*}{$\begin{array}{l}\text { Left } \\
\text { Rotation } \\
\text { Y-Plane }\end{array}$} & & Pre & Post & Post 2 & $\begin{array}{l}\text { a) pre }- \text { post } \\
\text { b) pre }- \text { post } 2\end{array}$ \\
\hline & $\mathrm{PO}$ & $10.5 \pm 3.6$ & $12.6 \pm 5.3$ & $9.7 \pm 2.3$ & a) $-2.1(-6.3$ to 2.0$) ; d=-0.46$ \\
\hline & MI & $12.3 \pm 5.1$ & $7.1 \pm 3.2$ & $8.6 \pm 2.8$ & $\begin{array}{l}\text { b) } 0.7(-2.1 \text { to } 3.6) ; d=0.26 \\
\text { a) } 5.1^{*}(1.0 \text { to } 9.3) ; d=1.18 \\
\text { b) } 3.6^{*}(0.7 \text { to } 6.5) ; d=0.84\end{array}$ \\
\hline & $\mathrm{AO}$ & $11.0 \pm 4.4$ & $5.1 \pm 3.3$ & $6.4 \pm 3.6$ & $\begin{array}{l}\text { a) } 5.8^{*}(1.6 \text { to } 10.0) ; d=1.71 \\
\text { b) } 4.5^{*}(1.7 \text { to } 7.4) ; d=1.25\end{array}$ \\
\hline
\end{tabular}

"pz.05; ${ }^{* *} p<.001 ;$ CI: confidence interval; SD: standard deviation; PO: placebo observation group; MI: motor imagery group; AO: action observation group. 


\section{Table 5 (on next page)}

Table 5. Within-group differences in right rotation cervical movement

${ }^{*} p<.05 ;{ }^{* *} p<.001 ; C l$ : confidence interval, SD: standard deviation; PO: placebo observation group; MI: motor imagery group; AO: action observation group. 
1 Table 5. Within-group differences in right rotation cervical movement

\begin{tabular}{|c|c|c|c|c|c|}
\hline Measure & Group & & Mean \pm SD & & Mean difference $(95 \%$ CI $)$; Effect size $(d)$ \\
\hline \multirow{4}{*}{$\begin{array}{c}\text { Right } \\
\text { Rotation } \\
\text { X-Plane }\end{array}$} & & Pre & Post & Post 2 & b) pre-post 2 \\
\hline & $\mathrm{PO}$ & $10.1 \pm 4.3$ & $11.0 \pm 4.1$ & $10.4 \pm 5.1$ & $\begin{array}{l}\text { a) }-0.9(-3.8 \text { to } 2.0) ; d=-0.21 \\
\text { b) }-0.3(-3.6 \text { to } 3.0) ; d=-0.06\end{array}$ \\
\hline & MI & $9.6 \pm 3.6$ & $8.3 \pm 3.8$ & $7.5 \pm 4.1$ & $\begin{array}{l}\text { a) } 1.2(-1.6 \text { to } 4.2) ; d=0.35 \\
\text { b) } 2.1(-1.2 \text { to } 5.4) ; d=0.54\end{array}$ \\
\hline & $\mathrm{AO}$ & $10.3 \pm 5.6$ & $6.7 \pm 4.5$ & $9.8 \pm 5.4$ & $\begin{array}{l}\text { a) } 3.6^{*}(0.7 \text { to } 6.5) ; d=0.72 \\
\text { b) } 0.5(-2.7 \text { to } 3.8) ; d=0.09\end{array}$ \\
\hline Measure & Group & & Mean \pm SD & & Mean difference $(95 \% \mathrm{CI})$; Effect size $(d)$ \\
\hline \multirow{4}{*}{$\begin{array}{c}\text { Right } \\
\text { Rotation } \\
\text { Y-Plane }\end{array}$} & & Pre & Post & Post 2 & $\begin{array}{l}\text { a) pre - post } \\
\text { b) pre - post } 2\end{array}$ \\
\hline & $\mathrm{PO}$ & $9.5 \pm 3.3$ & $7.5 \pm 2.7$ & $10.4 \pm 4.0$ & $\begin{array}{l}\text { a) } 2.0(-2.3 \text { to } 6.4) ; d=0.66 \\
\text { b) }-0.8(-5.2 \text { to } 3.5) ; d=-0.24\end{array}$ \\
\hline & MI & $9.8 \pm 4.4$ & $7.4 \pm 2.6$ & $8.9 \pm 3.4$ & $\begin{array}{l}\text { a) } 2.4(-1.9 \text { to } 6.7) ; d=0.66 \\
\text { b) } 0.9(-3.4 \text { to } 5.2) ; d=0.22\end{array}$ \\
\hline & $\mathrm{AO}$ & $11.7 \pm 5.4$ & $5.9 \pm 3.7$ & $7.3 \pm 3.8$ & $\begin{array}{l}\text { a) } 5.8^{*}(1.5 \text { to } 10.2) ; d=1.24 \\
\text { b) } 4.4^{*}(0.1 \text { to } 8.8) ; d=0.94\end{array}$ \\
\hline
\end{tabular}

"pz.05; ${ }^{* *} p<.001 ;$ CI: confidence interval, SD: standard deviation; PO: placebo observation group; MI: motor imagery group; AO: action observation group. 
Figure 1

\section{Study flow chart}

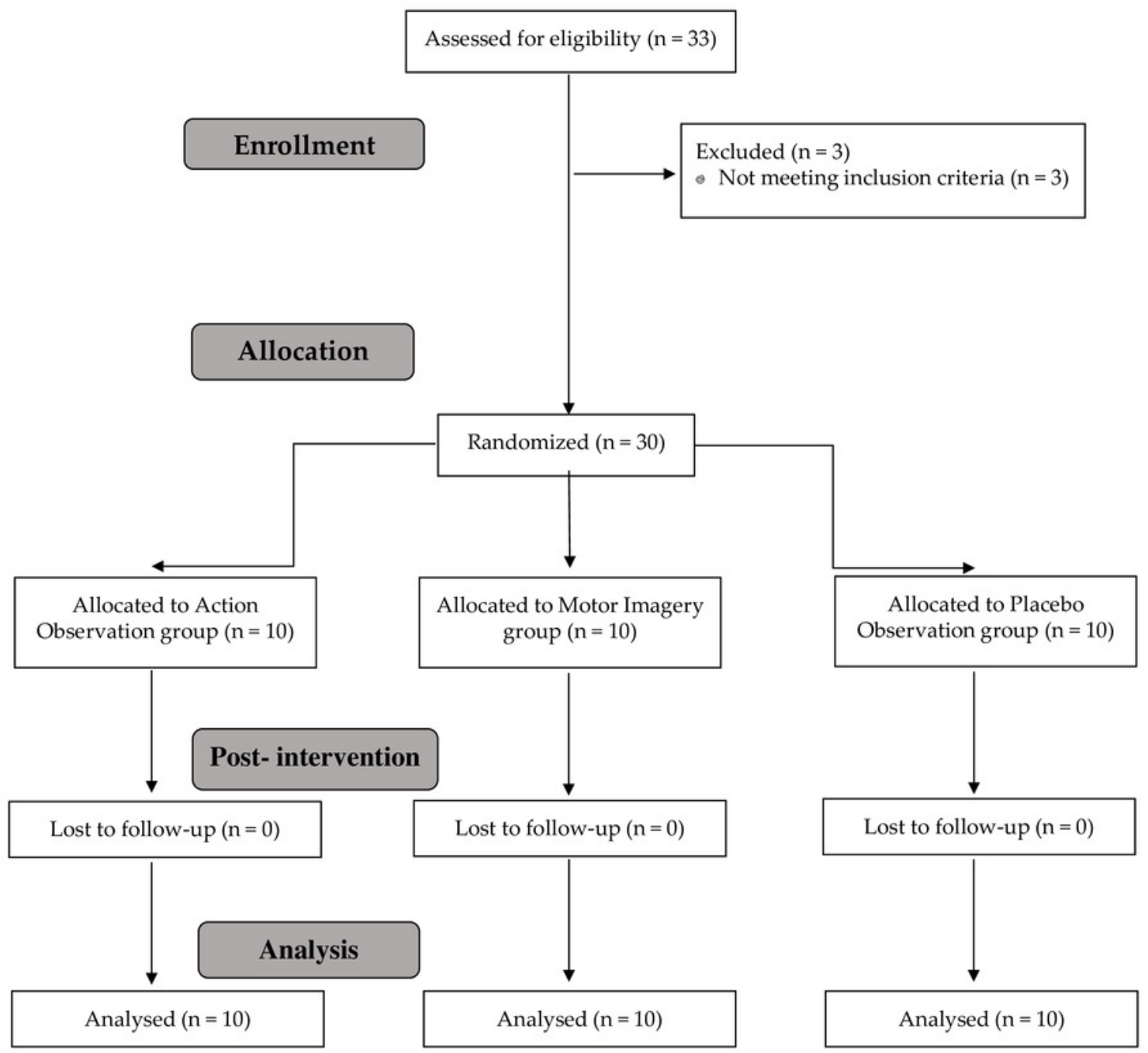


Figure 2

Protocol of the intervention

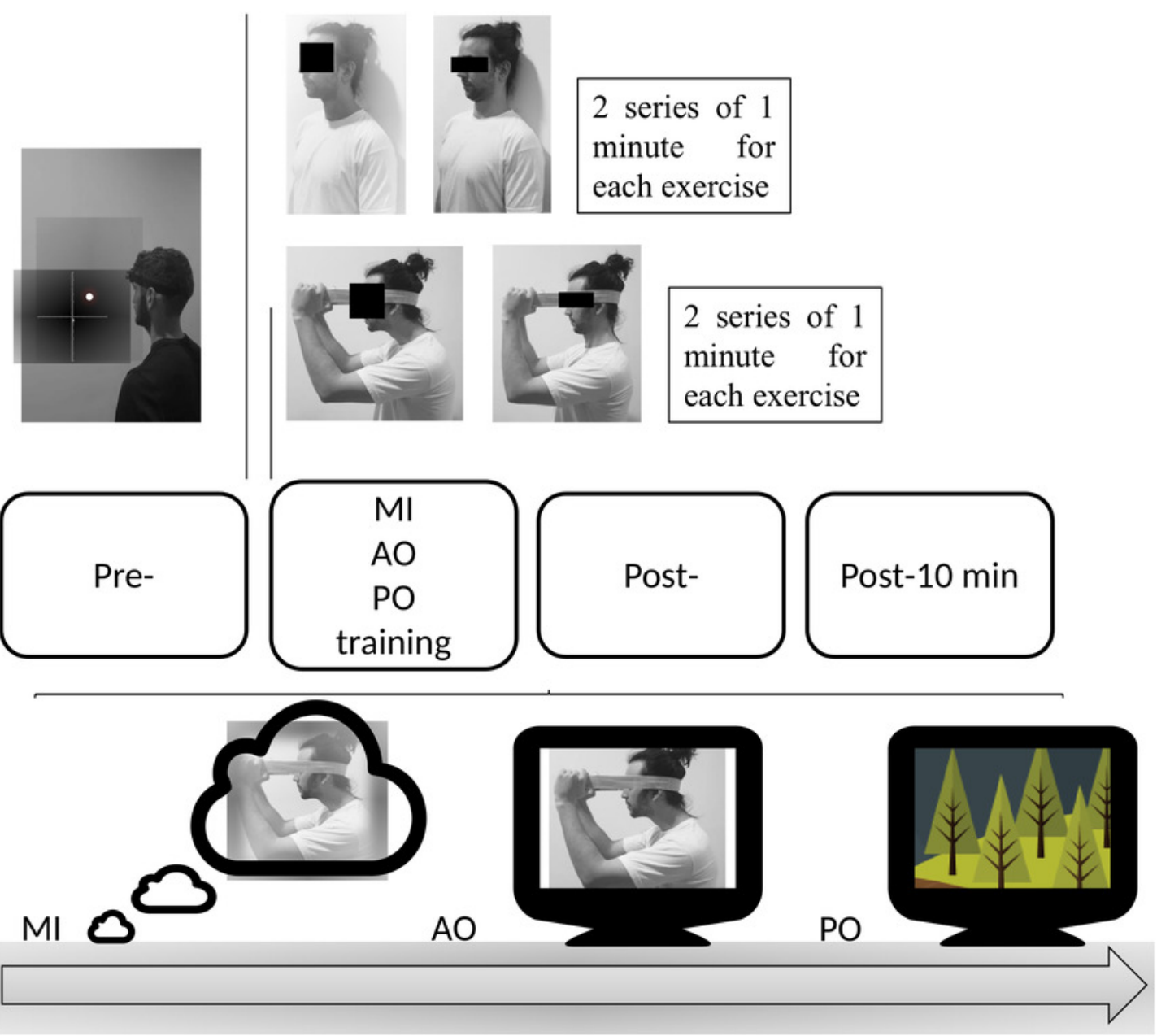


Figure 3

Between-group differences in flexo-extension cervical movements (Y-plane)

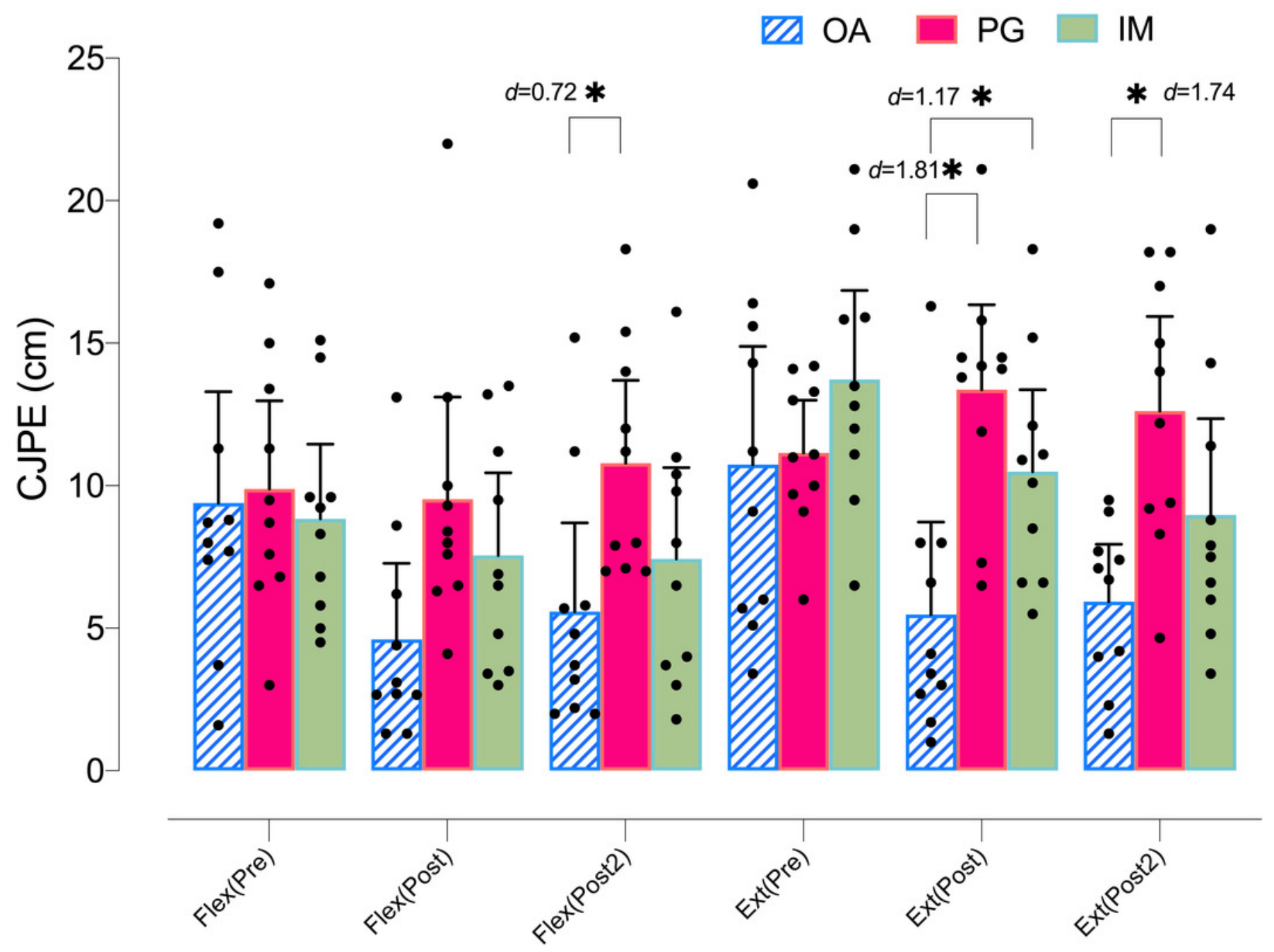


Figure 4

Between-group differences in rotation cervical movements (X-plane)

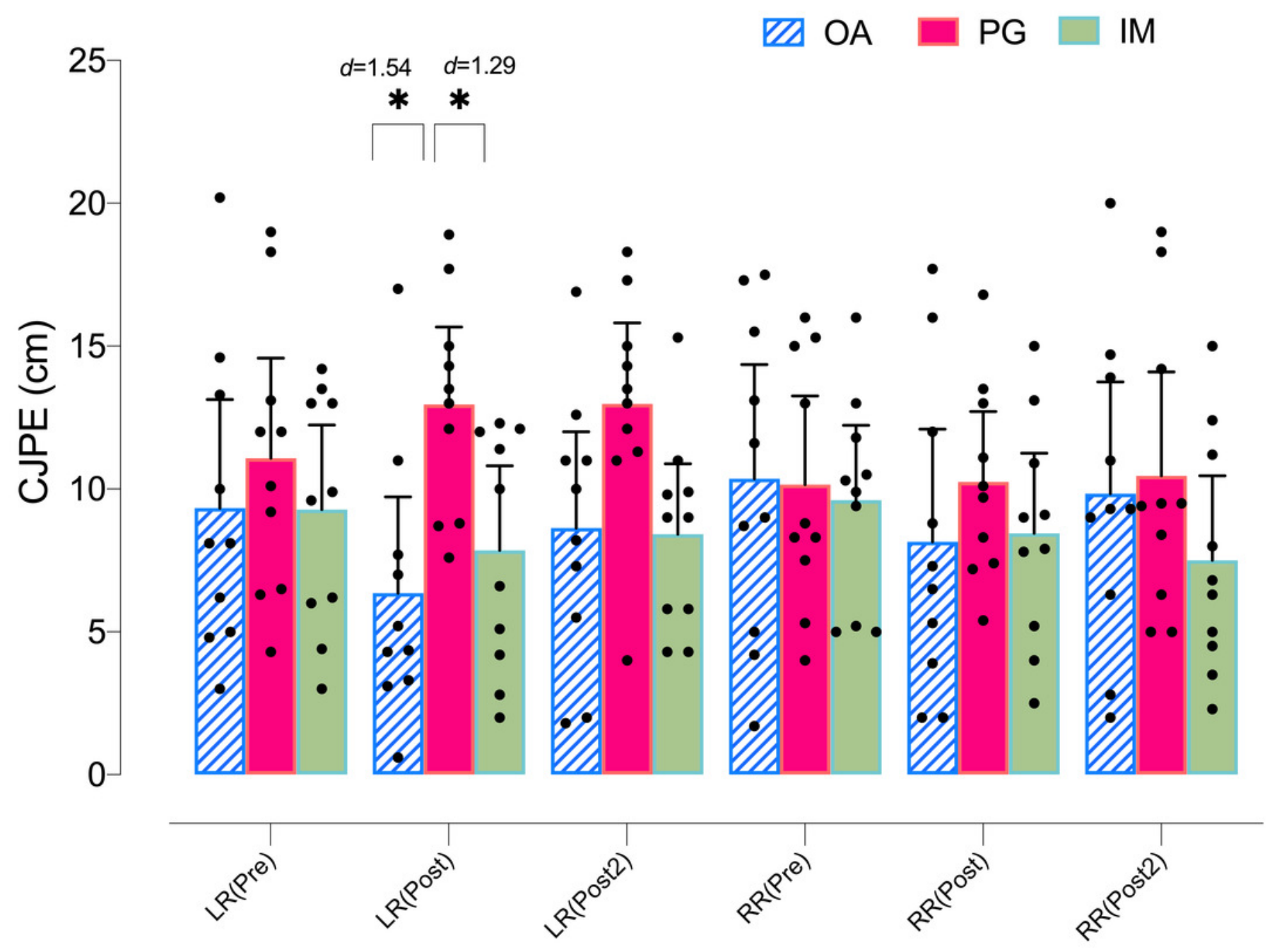


Figure 5

Between-group differences in rotation cervical movements (Y-plane)

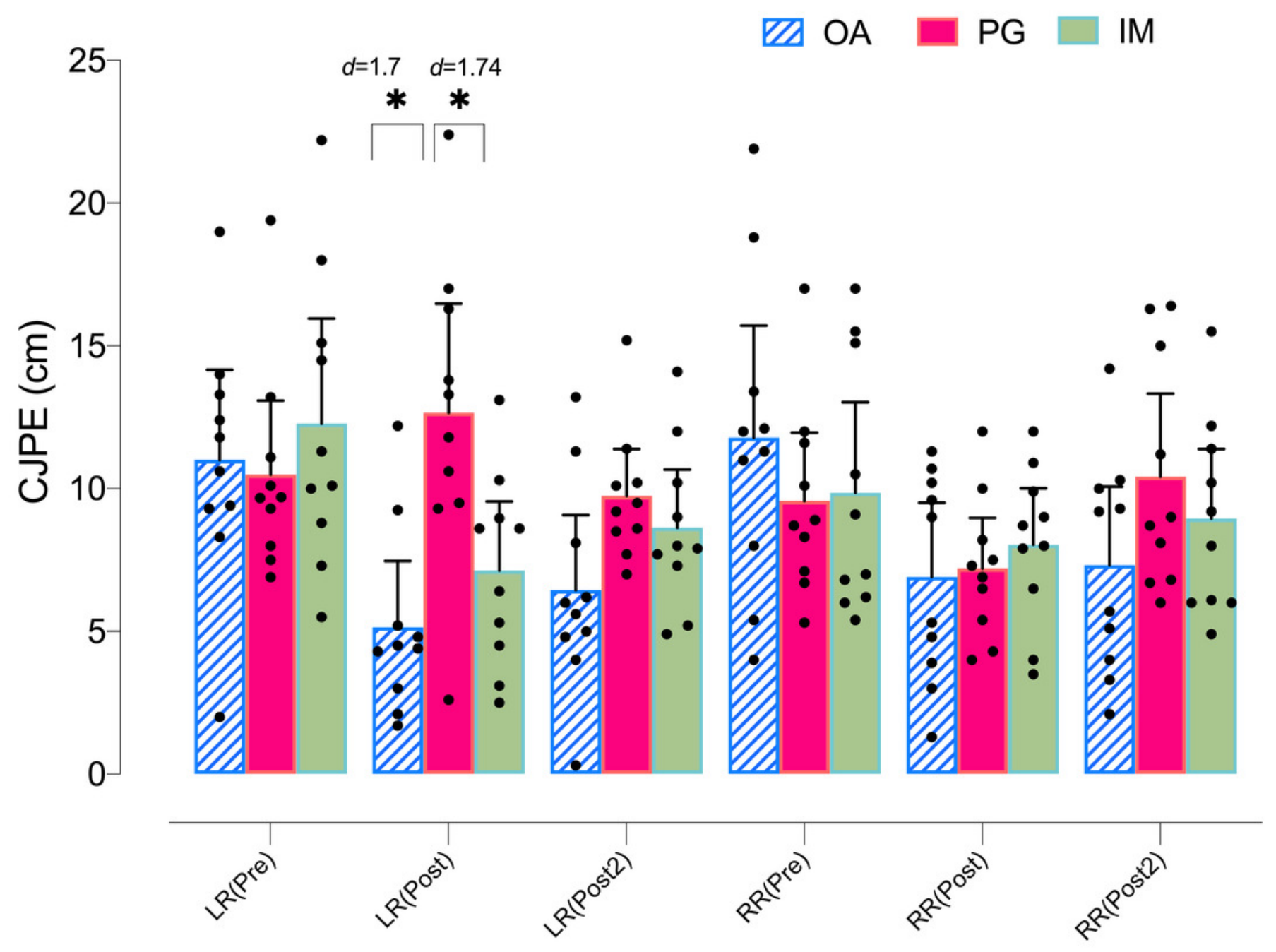

